\title{
Generalized Lagging Response of Thermoelastic Beams
}

\author{
Ibrahim H. El-Sirafy, ${ }^{1}$ Mohamed A. Abdou, ${ }^{2}$ and Emad Awad ${ }^{2}$ \\ ${ }^{1}$ Department of Mathematics, Faculty of Science, Alexandria University, Alexandria, Egypt \\ ${ }^{2}$ Department of Mathematics, Faculty of Education, Alexandria University, Alexandria, Egypt
}

Correspondence should be addressed to Emad Awad; emadawad78@alexu.edu.eg

Received 24 October 2013; Accepted 23 March 2014; Published 30 April 2014

Academic Editor: Oleg V. Gendelman

Copyright (c) 2014 Ibrahim H. El-Sirafy et al. This is an open access article distributed under the Creative Commons Attribution License, which permits unrestricted use, distribution, and reproduction in any medium, provided the original work is properly cited.

\begin{abstract}
The vibrations of Euler-Bernoulli metal beam are accommodated in the present study by taking into account the possibility of activating the microstructural effects, captured by the temperature gradient phase lag, in the fast transient process captured by the heat flux phase lag. The thermal moment is approximated as the difference between the top and the bottom surface temperatures (Massalas and Kalpakidis 1983). Three generalizations of the Biot model of thermomechanics are considered: Lord-Shulman, dualphase-lag (Tzou 1997), and modified dual-phase-lag (Awad 2012). It is found that when the response time is shortened, the material dimensions are small, or when the method of heating is changed, the dual-phase-lag model records a significant decrease in the lattice variables. The spurious serrations of the classical thermoelastic wave are smoothed in the dual-phase-lag wave. The dualphase-lag thermoelasticity is the closest macroscopic approach to the ultrafast model (Chen et al. 2002).
\end{abstract}

\section{Introduction}

The heat transport in metals, on the macroscopic level, can be modeled mathematically via the classical Fourier law for heat conduction:

$$
\vec{q}(\vec{r}, t)=-k \nabla T(\vec{r}, t),
$$

where $\vec{q}$ is the heat flux vector, $\vec{r}$ is the position vector, $t$ is the time, $k$ is the thermal conductivity, $T$ is the absolute temperature, and $\nabla$ denotes the spatial gradient operator.

When the problem of heat transfer at relatively small values of time is considered, it is found that the classical Fourier law (a) results in a nonzero temperature values for large values of distance, which contradicts the experimental results. For solving this paradox in the Fourierian model, Morse and Feshbach [1], Cattaneo [2], and Vernotte [3, 4] hypothesized the existence of thermal relaxation during the temperature propagation and expressed their hypothesis through the non-Fourierian law:

$$
\vec{q}(\vec{r}, t)+\tau_{0} \frac{\partial \vec{q}(\vec{r}, t)}{\partial t}=-k \nabla T(\vec{r}, t),
$$

where $\tau_{0}$ is a characteristic constant expressing the thermal relaxation time in the energy carriers collision and $\partial / \partial t$ is the differentiation with respect to the time variable $t$. The energy equation, yielded from $(b)$, is of the hyperbolic type that predicts a wave nature for temperature propagation with finite speed; see Joseph and Preziosi [5] and Tzou [6].

Based on the non-Fourier law (b), Lord and Shulman [7] established a linear theory of isotropic thermoelastic materials, Lord-Shulman model, to replace the classical one due to Biot [8]. Sherief and his colleagues [9-11] have taken into account the Lord-Shulman model in details, and more informative reviews can be found in Chandrasekharaiah [12], Hetnarski and Ignaczak [13], and Ignaczak and OstojaStarzewski [14].

On the microscopic level, because of the rapid development and growing interests of the short-pulse laser (laser with pulse duration ranging from $10^{-9}$ to $10^{-15}$ seconds), great efforts were theoretically and experimentally directed to evaluate the energy exchange between electrons and phonons during laser heating process and introduce a governing law which measures the electron temperature and lattice temperature; see [15-19]. When the response time is short and the material thickness is small so that it becomes comparable with the atomic distances in the scale sense, the non-Fourier law $(b)$ fails to predict the correct experimental 
values; see Qiu and Tien [20, 21]. As a result, a novel law of heat transfer was proposed to replace the Cattaneo-Vernotte equation $(b)$ and the conventional energy equation $-\partial q / \partial x=$ $\rho c_{v}(\partial T / \partial t)$; see Anisimov et al. [16]:

$$
\begin{gathered}
C_{e}\left(T_{e}\right) \frac{\partial T_{e}}{\partial t}=\nabla \cdot\left(k \nabla T_{e}\right)-G\left(T_{e}-T_{l}\right)+S \\
C_{l}\left(T_{l}\right) \frac{\partial T_{l}}{\partial t}=G\left(T_{e}-T_{l}\right),
\end{gathered}
$$

where $C_{e}$ and $C_{l}$ are the heat capacities of electron and lattice, respectively, $T_{e}$ and $T_{l}$ are the electron and lattice absolute temperatures, respectively, $S$ is the laser source term, and $G$ denotes the electron-phonon coupling factor. The system $(c)$ was phenomenological not based on a rigorous thermodynamical basis. Qiu and Tien [21] introduced a thermodynamic derivation for the phenomenological system (c) and its hyperbolic version.

Till 1995, all the works mentioned above were confined to the knowledge of specialists of Statistical Mechanics and Quantum Mechanics. Tzou [23, 24] introduced the lagging behavior idea in order to incorporate the microscopic interactions into the macroscopic formulae and, hence, to involve as many researchers as possible in the rapid growth of microscale heat transfer achieved in this time. The generalized lagging behavior (generalized dual-phaselag, or DPL) is briefly proposing a non-Fourier law of heat conduction which replaces $(a)$ and $(b)$ and takes the form

$$
\vec{q}\left(\vec{r}, t+\tau_{q}\right)=-k \nabla T\left(\vec{r}, t+\tau_{T}\right),
$$

where $\tau_{q}$ and $\tau_{T}$ are, respectively, the heat flux and the temperature gradient phase lags. When the Taylor expansion is used with neglecting the terms $O\left(\tau_{q}^{3}\right)$ and $O\left(\tau_{T}^{2}\right),(d)$ reads

$$
\left(1+\tau_{q} \frac{\partial}{\partial t}+\frac{\tau_{q}^{2}}{2} \frac{\partial^{2}}{\partial t^{2}}\right) \vec{q}(\vec{r}, t)=-k\left(1+\tau_{T} \frac{\partial}{\partial t}\right) \nabla T(\vec{r}, t)
$$

where combining $(e)$ with the energy equation $-\partial q / \partial x=$ $\rho c_{v}(\partial T / \partial t)$ yields the same equation governing the lattice temperature and resulted from the hyperbolic version of $(c)$; refer also to Tzou and $\mathrm{Xu}$ [25].

Awad [26] has proposed a refined aspect to $(e)$ to be on the form

$$
\left(1+\tau_{q} \frac{\partial}{\partial t}+\tau_{m}^{2} \frac{\partial^{2}}{\partial t^{2}}\right) \vec{q}(\vec{r}, t)=-k\left(1+\tau_{T} \frac{\partial}{\partial t}\right) \nabla T(\vec{r}, t),
$$

where $\tau_{m}^{2} \neq 0.5 \tau_{q}^{2}$. In $(d)-(f), \tau_{T}$ captures the microstructural effects $\left(C_{l} / G\right.$ ), and $\tau_{q}^{2}$ (or $\tau_{m}^{2}$ ) captures the ballistic behavior of electron transport [25].

Tzou [27] and Chandrasekharaiah [12], based on (e), have derived the linear theory of dual-phase-lag thermoelasticity, DPLT, that has been also reviewed in the survey due to Hetnarski and Ignaczak [13]. In [26], another version of
DPLT has been proposed using the law $(f)$; we will refer to this version as MDPLT. The non-Fourier law $(e)$ has been extended to thermoelectric materials by Abdou et al. [28].

The coupled equations of thermoelastic beams, including the effects of shear deformation and rotatory inertia, were derived by Jones [29]. Jones [29] referred to the paper of Ignaczak and Nowacki [30] that presented a similar analysis; however, he did not supply any appraisal to the work because the reference was not available. Massalas and Kalpakidis [31] introduced an analytical solution to a simply supported Euler-Bernoulli beam without rotatory inertia. They expressed the thermal moment as a linear combination of the upper and bottom surface temperatures of the beam. Sankar and Tzeng [32] have analytically solved the thermoelastic equilibrium equation of a functionally graded EulerBernoulli beam, by generalizing Sankar [33] work. They suggested that the temperature changes exponentially through the beam thickness. Babaei et al. [34] have numerically solved the coupled thermoelastic equations of a functionally graded Euler-Bernoulli beam by employing the Galerkin finite element method [35]. They made use of a combination of the ideas proposed by $[31,32]$ to express the temperature. Sun et al. $[22,36]$ have studied the laser-induced vibrations of a generalized thermoelastic Euler-Bernoulli beam. They adopted the Lord-Shulman model in their analysis and followed the Massalas-Kalpakidis assumption [31] with sinusoidal representation of the temperature through the thickness of the beam obeying zero-temperature gradient on its upper and bottom surfaces. Khisaeva and Ostoja-Starzewski [37] have studied the thermoelastic damping of a generalized Euler-Bernoulli microscale beam under the Lord-Shulman model. Youssef and Elsibai [38-41] have explored the vibration of Euler-Bernoulli gold nanobeam due to ramp type heating on one end of the beam under various theories of thermoelasticity. They adopted the same assumption of [22]. We refer to some analytical methods that have been introduced by el-Sirafy and Abdou [42] and Abdou and Aseeri [43].

It appears that when the ultrashort pulsed laser and the micro/nanostructures are collected in one subject, it is quite natural to give a great deal to the microstructural effects during the fast-transient process. Motivated by the above works, we will reconsider the micro/nanostructural Euler-Bernoulli beams responses in this study by taking into account the DPLT/MDPLT models. The problem will be mathematically modeled in the next section. Two different methods of heating will be considered on the upper surface of the beam: ultrashort pulsed laser heating and constant heat flux. The solutions will be derived in the third section by using Laplace transform and the direct approach techniques. A numerical technique will be adopted in the fourth section to recover the solutions in the physical domain, and then a microscale gold beam is chosen to study the essential differences between the mathematical models and the heating methods. The lattice variables are plotted against the spatial and temporal variables to show the way in which these variables decay and the domain in which the heat influences; see [44-47]. 


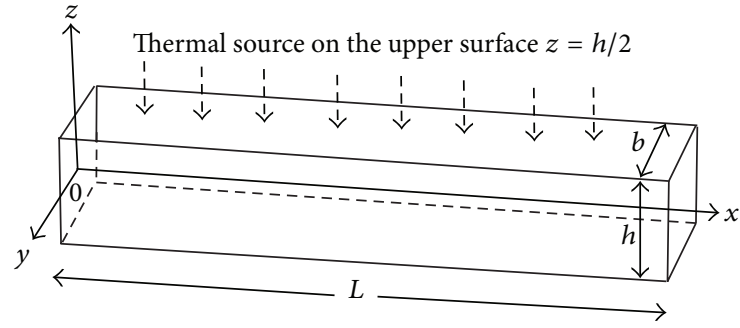

FIGURE 1: Problem description.

\section{Problem Modeling}

Consider an isotropic homogeneous rectangular thermoelastic beam of length $(0 \leq x \leq L)$, width $(-b / 2 \leq y \leq b / 2)$, and thickness $(-h / 2 \leq z \leq h / 2)$, where $x, y$, and $z$ are the usual Cartesian axes lying, respectively, along the length, width, and thickness of the beam so that $x$-axis coincides with the beam axis and $y$ and $z$ axes coincide with the end $(x=0)$ with origin located at the axis of the beam; see Figure 1 .

According to the Euler-Bernoulli assumption, see Jones [29] and Hetnarski and Eslami [48], we assume that the displacement components are given by

$$
\begin{gathered}
u(x, y, z, t)=-z \frac{\partial w}{\partial x} \quad v(x, y, z, t)=0 \\
w(x, y, z, t)=w(x, t),
\end{gathered}
$$

where $w(x, t)$ is the lateral deflection of the beam. From now on, all the variables are assumed to be independent of $y$.

The (axial) stress along the beam axis, $\sigma$, is given by the modified Hooke's law:

$$
\sigma=E\left(\varepsilon_{x}-\frac{\alpha_{T}}{1-2 \nu} \theta\right),
$$

where $E$ is the Young modulus, $\alpha_{T}$ is the coefficient of linear thermal expansion, $v$ is the Poisson ratio, $\varepsilon_{x}=\partial u / \partial x$ is the dilatation along the beam axis, and $\theta(x, z, t)=T-T_{0}$ denotes the deviation of absolute temperature $T(x, z, t)$ from the ambient temperature $T_{0}$.

The bending moment resultant of the beam, $M$, can be evaluated via the following relation:

$$
M=-\int_{-h / 2}^{h / 2} \sigma b z \mathrm{~d} z
$$

Making use of Euler-Bernoulli assumption (1), $\varepsilon_{x}$ and Hooke's law (2) in (3), we arrive at

$$
\begin{gathered}
\sigma=-\left(E z \frac{\partial^{2} w}{\partial x^{2}}+\beta \theta\right) \\
M=E I\left(\frac{\partial^{2} w}{\partial x^{2}}+b M_{T}\right),
\end{gathered}
$$

where $\beta=E \alpha_{T} /(1-2 v), I$ is the second moment of the crosssection area of the beam and $M_{T}$ is the thermal moment, and $I$ and $M_{T}$ are given by

$$
I=\int_{-h / 2}^{h / 2} b z^{2} \mathrm{~d} z=\frac{b h^{3}}{12}, \quad M_{T}=\frac{\alpha_{T}}{I(1-2 \nu)} \int_{-h / 2}^{h / 2} \theta z \mathrm{~d} z .
$$

The equation governing transverse deflections of the beam, neglecting the rotatory inertia effects, is [29]

$$
\frac{\partial^{2} M}{\partial x^{2}}+\rho A \frac{\partial^{2} w}{\partial t^{2}}=0
$$

where $\rho$ denotes the beam density and $A=b h$ is the crosssectional area of the beam.

In combining (4b) and (5), we have

$$
\frac{\partial^{4} w}{\partial x^{4}}+b \frac{\partial^{2} M_{T}}{\partial x^{2}}+\frac{\rho A}{E I} \frac{\partial^{2} w}{\partial t^{2}}=0 .
$$

In addition to (6), the energy equation including the hyperbolic theories, considered in [26], is given as

$$
\begin{aligned}
k\left(1+\tau_{T} \frac{\partial}{\partial t}\right) \nabla^{2} \theta= & {\left[1+\tau_{q} \frac{\partial}{\partial t}+\left(n_{1} \tau_{m}^{2}+n_{2} \frac{\tau_{q}^{2}}{2}\right) \frac{\partial^{2}}{\partial t^{2}}\right] } \\
& \times\left(\rho c_{v} \frac{\partial \theta}{\partial t}+\beta T_{0} \frac{\partial \varepsilon_{x}}{\partial t}\right) \\
& -\left(1+n_{1} \tau_{q_{2}} \frac{\partial}{\partial t}+\left(n_{2}+n_{3}\right) \tau_{q} \frac{\partial}{\partial t}\right) S_{i},
\end{aligned}
$$

where $\nabla^{2}=\left(\partial^{2} / \partial x^{2}\right)+\left(\partial^{2} / \partial z^{2}\right)$ is the two-dimensional Laplace operator, $\tau_{q}=\tau_{q 1}+\tau_{q 2}, \tau_{m}=\sqrt{\tau_{q 1} \tau_{q 2}}, \tau_{q 1}=$ $(1 / G)\left(\left(1 / C_{l}\right)+\left(1 / C_{e}\right)\right)^{-1}, \tau_{q 2}=\tau_{F}, \tau_{F}$ is the relaxation time at the Fermi level, $c_{v}$ is the specific heat at constant volume, $S_{i}=S_{i}(z, t)$ denotes the initial heating source, to be defined later, and $n_{1}, n_{2}$, and $n_{3}$ are controlling numbers which take their values from the set $\{0,1\}$.

Equation (7), in essence, is considered a unified energy equation in the linear theory of thermoelasticity with finite wave speed. Although it involves various thermoelastic theories, we will focus on the following cases.

(i) When $n_{1}=n_{2}=0, n_{3}=1, \tau_{T}=0$, (7) reduces the energy equation of the Lord-Shulman thermoelasticity [7]. This model has been employed to microscale beams by Sun et al. [22,36].

(ii) When $n_{1}=n_{3}=0, n_{2}=1$, (7) reduces the energy equation of the hyperbolic dual-phase-lag thermoelasticity, DPLT, [12, 27].

(iii) When $n_{2}=n_{3}=0, n_{1}=1$, (7) reduces the energy equation of the modified hyperbolic dual-phase-lag thermoelasticity, MDPLT, [26].

In view of the Euler-Bernoulli assumption (1), (7) could take the following form:

$$
\gamma_{1} \nabla^{2} \theta=\gamma_{2}\left(\frac{\rho c_{v}}{k} \frac{\partial \theta}{\partial t}-\frac{\beta T_{0}}{k} z \frac{\partial^{3} w}{\partial x^{2} \partial t}\right)-S
$$


where

$$
\begin{gathered}
S=S(z, t)=\gamma_{3} S_{i}(z, t), \quad \gamma_{1}=1+\tau_{T} \frac{\partial}{\partial t}, \\
\gamma_{2}=1+\tau_{q} \frac{\partial}{\partial t}+n_{1} \tau_{m}^{2} \frac{\partial^{2}}{\partial t^{2}}+n_{2} \frac{\tau_{q}^{2}}{2} \frac{\partial^{2}}{\partial t^{2}}, \\
\gamma_{3}=\frac{1}{k}\left(1+n_{1} \tau_{q_{2}} \frac{\partial}{\partial t}+\left(n_{2}+n_{3}\right) \tau_{q} \frac{\partial}{\partial t}\right) .
\end{gathered}
$$

Following Massalas and Kalpakidis [31], we multiply (8a) by $z \mathrm{~d} z$ and integrate over the interval $(-h / 2, h / 2)$; this leads to

$$
\begin{aligned}
\gamma_{1}\{( & \left.\frac{\partial^{2} M_{T}}{\partial x^{2}}-\frac{1}{h^{2}} M_{T}\right)(x, t)+\frac{\alpha_{T} h}{2 I(1-2 v)} \\
\times & {\left.\left[\frac{\partial \theta}{\partial z}\left(x, \frac{h}{2}, t\right)+\frac{\partial \theta}{\partial z}\left(x,-\frac{h}{2}, t\right)\right]\right\} } \\
= & \gamma_{2}\left(\frac{\rho c_{v}}{k} \frac{\partial M_{T}}{\partial t}-\frac{E T_{0} \alpha_{T}^{2}}{k b(1-2 v)^{2}} \frac{\partial^{3} w}{\partial x^{2} \partial t}\right) \\
& \times(x, t)-S_{h}(t),
\end{aligned}
$$

where $M_{T}$ is mathematically approximated as the difference between the temperatures at the upper and bottom surfaces of the beam, or, alternatively, the temperature is assumed to vary linearly through the thickness of the beam, thus, we have

$$
\theta\left(x, \frac{h}{2}, t\right)-\theta\left(x,-\frac{h}{2}, t\right)=\frac{I(1-2 v)}{\alpha_{T} h^{2}} M_{T}(x, t)
$$

and $S_{h}(t)$ is given by

$$
S_{h}(t)=\frac{\alpha_{T}}{I(1-2 \nu)} \int_{-h / 2}^{h / 2} z S(z, t) \mathrm{d} z .
$$

(Assumption (9b) was successfully used by Massalas and Kalpakidis [31] for beams with infinitesimal thickness.)

The problem, so far, is not emphasized in the small-scale length. Introducing of the following dimensionless quantities seems to be crucial for the paper aim:

$$
\begin{aligned}
\grave{x}=\frac{x}{L}, \quad \grave{w} & =\frac{w}{L}, \quad \grave{t}=\frac{v t}{L}, \quad \grave{M}_{T}=L^{2} M_{T}, \\
\grave{\sigma} & =\frac{\sigma}{E}, \quad v=\sqrt{\frac{E}{\rho} .}
\end{aligned}
$$

Accordingly, (6) and (9a), (9b), and (9c) read, after dropping the dashes for convenience,

$$
\frac{\partial^{4} w}{\partial x^{4}}+r_{2} \frac{\partial^{2} M_{T}}{\partial x^{2}}+\frac{12}{r_{1}^{2}} \frac{\partial^{2} w}{\partial t^{2}}=0
$$

$$
\begin{aligned}
\Gamma_{1}\{( & \left.\frac{\partial^{2} M_{T}}{\partial x^{2}}-\frac{1}{r_{1}^{2}} M_{T}\right)(x, t) \\
& \left.+c_{1}\left[\frac{\partial \theta}{\partial z}\left(x, \frac{h}{2}, t\right)+\frac{\partial \theta}{\partial z}\left(x,-\frac{h}{2}, t\right)\right]\right\} \\
& =\Gamma_{2}\left(c_{2} \frac{\partial M_{T}}{\partial t}-c_{3} \frac{\partial^{3} w}{\partial x^{2} \partial t}\right)(x, t)-L^{4} S_{h}^{*}(t),
\end{aligned}
$$

where

$$
\begin{gathered}
r_{1}=\frac{h}{L}, \quad r_{2}=\frac{b}{L}, \quad c_{1}=\frac{6 \alpha_{T} L}{r_{1}^{2} r_{2}(1-2 v)}, \\
c_{2}=\frac{\rho c_{v} L v}{k}, \quad c_{3}=\frac{E T_{0} \alpha_{T}^{2} L v}{k r_{2}(1-2 v)^{2}}, \\
\Gamma_{1}=1+\xi_{1} \frac{\partial}{\partial t}, \quad \Gamma_{2}=1+\xi_{2} \frac{\partial}{\partial t}+\left(n_{1} \xi_{3}^{2}+n_{2} \frac{\xi_{2}^{2}}{2}\right) \frac{\partial^{2}}{\partial t^{2}}, \\
\xi_{1}=\frac{v \tau_{T}}{L}, \quad \xi_{2}=\frac{v \tau_{q}}{L}, \quad \xi_{3}=\frac{v \tau_{m}}{L}
\end{gathered}
$$

and $S_{h}^{*}(t)$ is the dimensionless form of $S_{h}(t)$. Thus, (11) are the governing equations of a vibrating Euler-Bernoulli beam with length $L$ and aspect ratios $r_{1}$ and $r_{2}$, under two transverse thermal causes: (i) temperature gradient on the upper and bottom surfaces and (ii) thermal source applied in a normal direction to the beam axis. Solving (11) leads to finding the beam lateral deflection $w$, the thermal moment $M_{T}$, and the axial stress $\sigma$. The thermal moment $M_{T}$ can be considered as having the same behavior of temperature.

\section{Solution in the Laplace Domain}

The direct approach was first applied to coupled thermoelastic problems by Bahar and Hetnarski [49]. It has recently showed successful results; see Ezzat and Awad $[50,51]$. The Laplace transform is defined by

$$
L\{f(t)\}=\int_{0}^{\infty} e^{-s t} f(t) \mathrm{d} t=\bar{f}(s) .
$$

By assuming zero initial conditions for $w, M_{T}$, and their rates $\left\{\partial w / \partial t, \partial M_{T} / \partial t, \partial^{2} M_{T} / \partial t^{2}, \partial^{2} w / \partial x^{2}, \partial^{3} w / \partial x^{2} \partial t\right.$, $\left.\partial^{4} w / \partial x^{2} \partial t^{2}\right\}$, and in view of (11) and (13), we obtain

$$
\begin{gathered}
\left(D^{4}+\frac{12 s^{2}}{r_{1}^{2}}\right) \bar{w}(x, s)=-r_{2} D^{2} \bar{M}_{T}(x, s) \\
\left(D^{2}-\alpha_{1}\right) \bar{M}_{T}(x, s)=-\alpha_{2} D^{2} \bar{w}(x, s)-\bar{Q}^{*}(s),
\end{gathered}
$$


where

$$
\begin{gathered}
D=\frac{d}{d x}, \quad \alpha_{1}(s)=\frac{1}{r_{1}^{2}}+\frac{c_{2} \alpha_{2}}{c_{3}} \quad \alpha_{2}(s)=\frac{c_{3} s \bar{\Gamma}_{2}}{\bar{\Gamma}_{1}}, \\
\bar{\Gamma}_{1}=1+\xi_{1} s, \quad \bar{\Gamma}_{2}=1+\xi_{2} s+\left(n_{1} \xi_{3}^{2}+n_{2} \frac{\xi_{2}^{2}}{2}\right) s^{2}, \\
\bar{Q}^{*}(s)=c_{1}\left[\frac{d \bar{\theta}}{d z}\left(x, \frac{h}{2}, s\right)+\frac{d \bar{\theta}}{d z}\left(x,-\frac{h}{2}, s\right)\right]+\frac{L^{4} \bar{S}_{h}^{*}(s)}{\bar{\Gamma}_{1}}
\end{gathered}
$$

and $\bar{S}_{h}^{*}(s)$ denotes the Laplace transform of the heating source $S_{h}^{*}(t)$.

The differential equation of the lateral deflection $\bar{w}$ and the thermal moment $\bar{M}_{T}$ are

$$
\left\{D^{6}-\ell D^{4}+m D^{2}-n\right\}\left[\frac{\bar{w}_{M}}{\bar{M}_{T}}\right]=\left[\begin{array}{c}
0 \\
-\frac{12 s^{2}}{r_{1}^{2}} \bar{Q}^{*}
\end{array}\right],
$$

where

$$
\ell(s)=\alpha_{1}+r_{2} \alpha_{2}, \quad m(s)=\frac{12 s^{2}}{r_{1}^{2}}, \quad n(s)=m \alpha_{1} .
$$

The differential equation governing the lateral deflection $\bar{w}$ can take the form

$$
\left(D^{2}-\lambda_{1}^{2}\right)\left(D^{2}-\lambda_{2}^{2}\right)\left(D^{2}-\lambda_{3}^{2}\right) \bar{w}=0
$$

where $\pm \lambda_{1}, \pm \lambda_{2}$, and $\pm \lambda_{3}$ are the characteristic roots of the equation:

$$
\lambda^{6}-\ell \lambda^{4}+m \lambda^{2}-n=0
$$

and satisfy the well-known relations:

$$
\begin{gathered}
\lambda_{1}^{2}+\lambda_{2}^{2}+\lambda_{3}^{2}=\ell \quad \lambda_{1}^{2} \lambda_{2}^{2}+\lambda_{2}^{2} \lambda_{3}^{2}+\lambda_{1}^{2} \lambda_{3}^{2}=m . \\
\lambda_{1}^{2} \lambda_{2}^{2} \lambda_{3}^{2}=n
\end{gathered}
$$

See (A.1) in the Appendix.

Then the lateral deflection is given by

$$
\bar{w}(x, s)=\sum_{i=1}^{3}\left(A_{i} e^{\lambda_{i} x}+B_{i} e^{-\lambda_{i} x}\right),
$$

where the coefficients $A_{i}$ and $B_{i}, i=1,2,3$, to be determined later, are depending on the Laplace variable $s$.

The thermal moment is given by

$$
\bar{M}_{T}(x, s)=\sum_{i=1}^{3}\left(\grave{A}_{i} e^{\lambda_{i} x}+\grave{B}_{i} e^{-\lambda_{i} x}\right)+\frac{\overline{\mathrm{Q}}^{*}}{\alpha_{1}},
$$

where $\grave{A}_{i}$ and $\grave{B}_{i}, i=1,2,3$, are depending on the Laplace variable $s$. If a relation is established between $\left\{\grave{A}_{i}, \grave{B}_{i}\right\}$ and $\left\{A_{i}, B_{i}\right\}$, it will be needed six boundary conditions in order to determine the unknowns $\left\{A_{i}, B_{i}\right\}$. Proceed in this direction and substitute (20) and (21) into (15), it yields

$$
\left(\begin{array}{c}
\grave{A}_{i} \\
\grave{B}_{i}
\end{array}\right)=\frac{\alpha_{2} \lambda_{i}^{2}}{\alpha_{1}-\lambda_{i}^{2}}\left(\begin{array}{c}
A_{i} \\
B_{i}
\end{array}\right), \quad i=1,2,3,
$$

which helps us to rewrite (21) to be

$$
\bar{M}_{T}(x, s)=\sum_{i=1}^{3} \frac{\alpha_{2} \lambda_{i}^{2}}{\alpha_{1}-\lambda_{i}^{2}}\left(A_{i} e^{\lambda_{i} x}+B_{i} e^{-\lambda_{i} x}\right)+\frac{\bar{Q}^{*}}{\alpha_{1}} .
$$

By holding similar arguments, the axial stresses can be written as

$$
\begin{aligned}
\bar{\sigma}_{\text {avg }}(x, s)= & r_{1} \sum_{i=1}^{3} \lambda_{i}^{2}\left(\frac{r_{2} \alpha_{2}}{12\left(\lambda_{i}^{2}-\alpha_{1}\right)}-1\right)\left(A_{i} e^{\lambda_{i} x}+B_{i} e^{-\lambda_{i} x}\right) \\
& -\frac{r_{1} r_{2}}{12 \alpha_{1}} \bar{Q}^{*}
\end{aligned}
$$

where

$$
\bar{\sigma}_{\text {avg }}(x, s)=\overline{\grave{\sigma}}\left(x, \frac{h}{2}, s\right)-\overline{\grave{\sigma}}\left(x,-\frac{h}{2}, s\right)
$$

is used and we recalled (4a), the approximation of thermal moment (9b), (10) and (13), and (20) and (23).

In order to determine the unknown coefficients $A_{i}$ and $B_{i}, i=1,2,3$, we need to specify the thermal influence $\bar{Q}^{*}(s)$. In the next section, we will discuss two different applications of thermal loads over the upper surface of the beam.

\section{Applications}

In this section, we consider the vibrations of a gold microscale beam, of length $L=0.1 \mu \mathrm{m}$ and aspect ratios $r_{1}=0.1, r_{2}=$ 0.05 , induced by two methods of heating:

(i) laser source with non-Gaussian temporal profile set along the upper surface $z=h / 2$,

(ii) constant heat flux normal to the upper surface $z=$ $h / 2$.

First, consider the following profile:

$$
P(t)=\frac{I_{0} t}{t_{p}} \exp \left(-\frac{t}{t_{p}}\right)
$$

where $t_{p}$ is the laser-pulse duration and $I_{0}$ is the laser-pulse intensity, namely, the total energy carried by the laser beam per unit cross-section and unit time. The profile (25) has successfully been studied in the recent literatures [22, 26, 27, $36,52]$, in the case of microscale beams with single thermal relaxation and microfilm with dual-phase-lag.

The laser source $S_{i}$, according to case (i) of the present section, can be therefore defined as

$$
S_{i}(z, t)=\frac{1-R}{\delta} P(t) \exp \left(\frac{z-(h / 2)}{\delta}\right),
$$




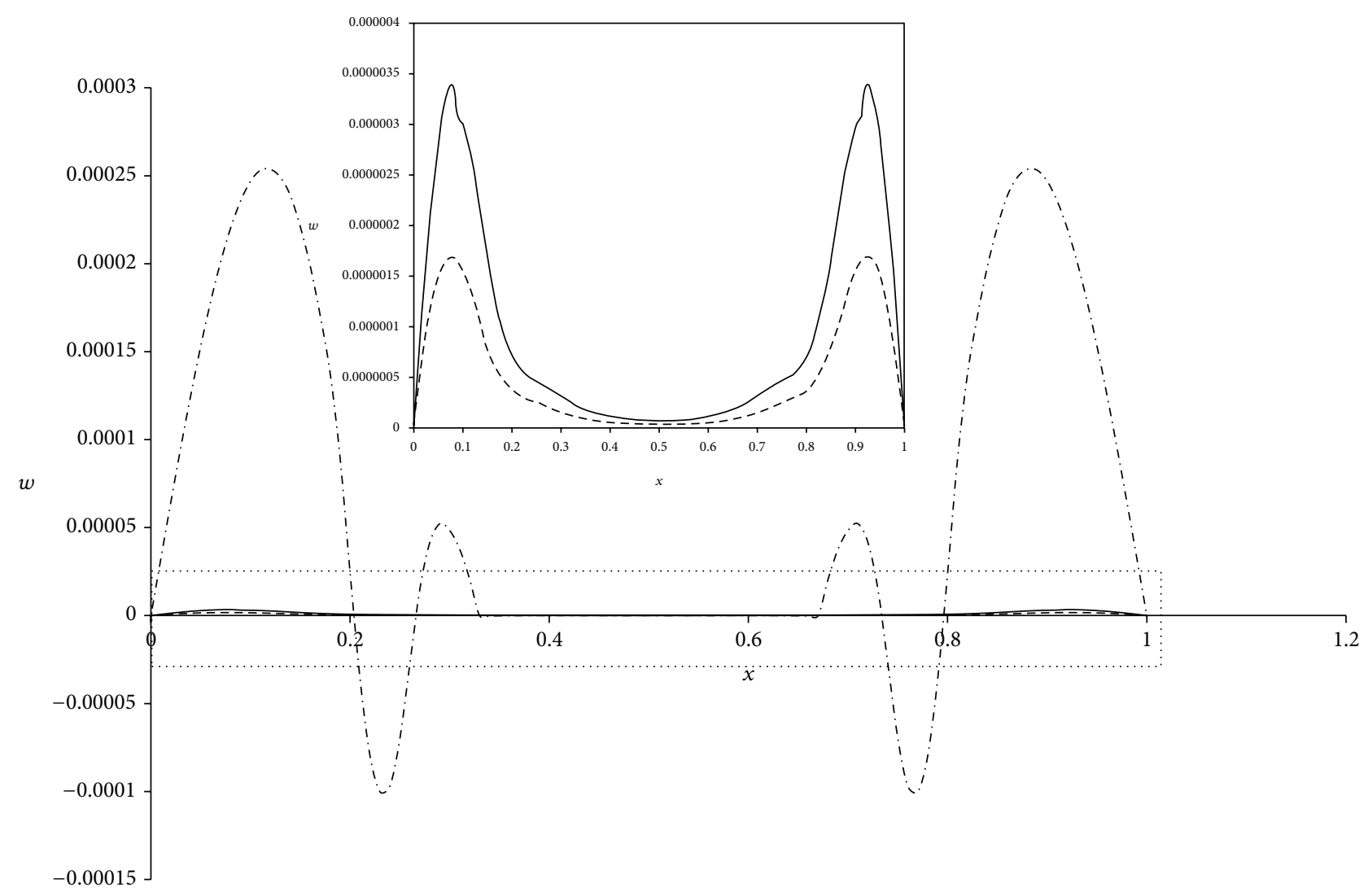

- DPLT

... L Lord-Shulman

- - MDPLT

FIGURE 2: Laser-induced lateral deflection at dimensionless time $t=0.1$ under the three models.

where $R$ and $\delta$ have their usual meaning, the surface reflectivity and the penetration depth of the beam material.

Furthermore, the beam is considered to be simply supported with ends ( $x=0, x=L)$ kept at the ambient temperature. Thereby, the boundary conditions are determined through

$$
\begin{array}{lll}
w(0, t)=0 & \frac{\partial^{2} w(0, t)}{\partial x^{2}}=0 & M_{T}(0, t)=0 \\
w(L, t)=0 & \frac{\partial^{2} w(L, t)}{\partial x^{2}}=0 & M_{T}(L, t)=0 .
\end{array}
$$

Moreover, the temperature gradient on the upper and bottom surfaces are assumed null (no heat losses); namely,

$$
\frac{\partial \theta}{\partial z}\left(x, \frac{h}{2}, t\right)=\frac{\partial \theta}{\partial z}\left(x,-\frac{h}{2}, t\right)=0
$$

Next, we can evaluate the thermal influence $\bar{Q}^{*}$ given by (16), and the result is

$$
\bar{Q}^{*}(s)=\frac{6(1-R) J_{0} a_{\delta} \xi_{5}\left[1+\left(n_{1} \xi_{4}+n_{2} \xi_{2}+n_{3} \xi_{2}\right) s\right]}{r_{1}^{2} r_{2}(1-2 v)\left(1+\xi_{5} s\right)^{2} \bar{\Gamma}_{1}},
$$

where

$$
\begin{gathered}
a_{\delta}=\left[\left(1-\frac{2 \delta}{h}\right)+\left(1+\frac{2 \delta}{h}\right) e^{-h / \delta}\right], \quad J_{0}=\frac{L \alpha_{T} I_{0}}{k}, \\
\xi_{4}=\frac{v \tau_{q_{2}}}{L}, \quad \xi_{5}=\frac{v t_{p}}{L} .
\end{gathered}
$$

The nondimensional forms of the boundary conditions (27a), (27b), and (27c) in the Laplace domain read

$$
\begin{array}{lll}
\bar{w}(0, s)=0 & \frac{\mathrm{d}^{2} \bar{w}(0, s)}{\mathrm{d} x^{2}}=0 & \bar{M}_{T}(0, s)=0 \\
\bar{w}(1, s)=0 & \frac{\mathrm{d}^{2} \bar{w}(1, s)}{\mathrm{d} x^{2}}=0 & \bar{M}_{T}(1, s)=0 .
\end{array}
$$

By the aid of (29a) and (29b) and the thermal influence (28a), and (28b), the solutions (20) and (23) lead to

$$
\sum_{i=1}^{3}\left(A_{i}+B_{i}\right)=0
$$




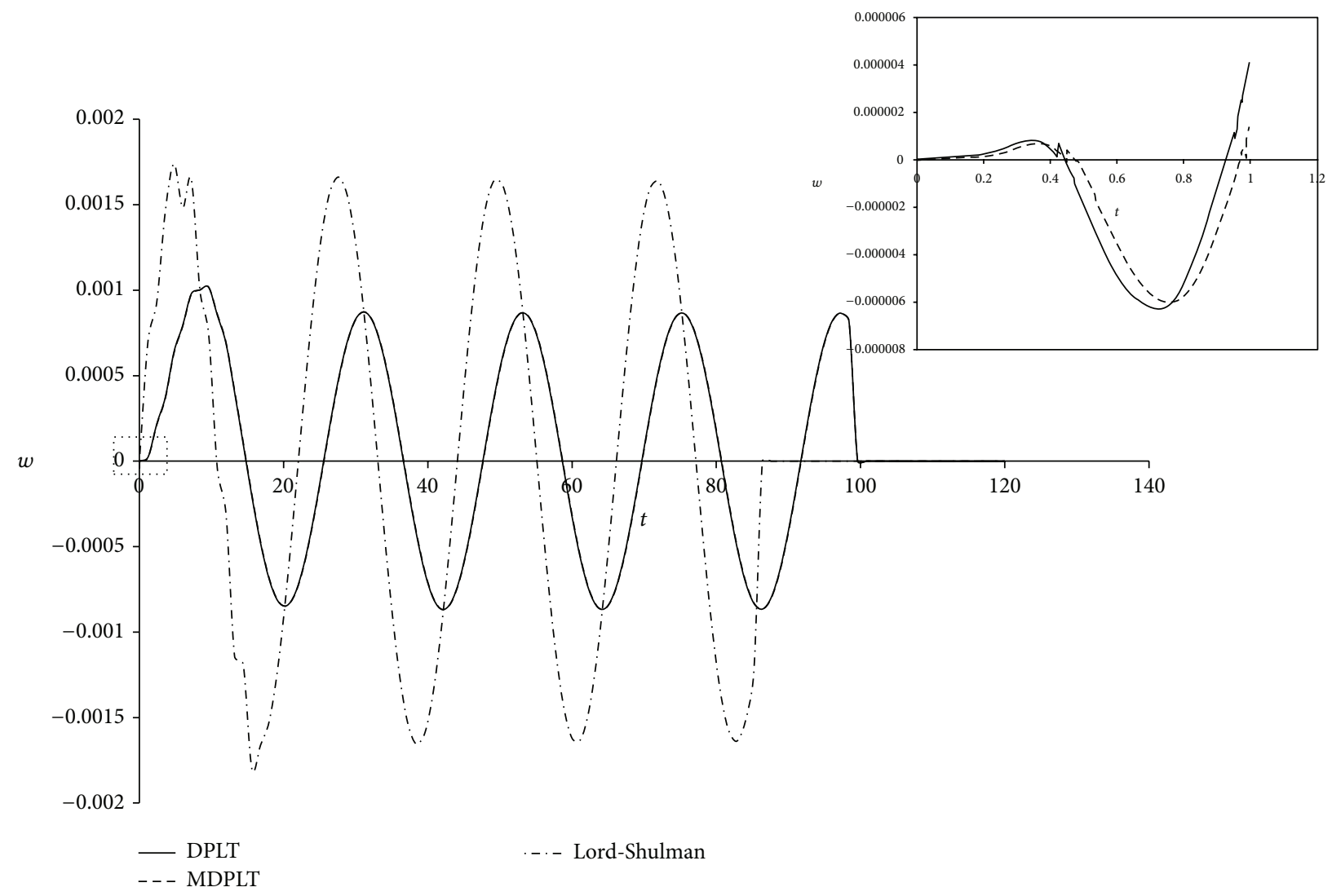

FIGURE 3: Laser-induced midpoint lateral deflection under the three models.

$$
\begin{gathered}
\sum_{i=1}^{3} \lambda_{i}^{2}\left(A_{i}+B_{i}\right)=0 \\
\sum_{i=1}^{3} \frac{\lambda_{i}^{2}}{\lambda_{i}^{2}-\alpha_{1}}\left(A_{i}+B_{i}\right)=\frac{\bar{Q}^{*}}{\alpha_{1} \alpha_{2}} \\
\sum_{i=1}^{3}\left(A_{i} e^{\lambda_{i}}+B_{i} e^{-\lambda_{i}}\right)=0 \\
\sum_{i=1}^{3} \lambda_{i}^{2}\left(A_{i} e^{\lambda_{i}}+B_{i} e^{-\lambda_{i}}\right)=0 \\
\sum_{i=1}^{3} \frac{\lambda_{i}^{2}}{\lambda_{i}^{2}-\alpha_{1}}\left(A_{i} e^{\lambda_{i}}+B_{i} e^{-\lambda_{i}}\right)=\frac{\bar{Q}^{*}}{\alpha_{1} \alpha_{2}} .
\end{gathered}
$$

Then, by solving (30a), (30b), (30c), (30d), (30e), and (30f) one can obtain the required coefficients; see (A.3) in the Appendix.

Second, as stated in assumption (ii), we replace the laser source term with a constant heat flux $\left\{-q_{0}\right\}$ normal to the upper surface of the beam, with keeping the bottom surface at zero temperature gradient. The boundary conditions on the upper and bottom surface are then given through the DPL law of heat conduction as

$$
q_{0}=k\left(1+\tau_{T} \frac{\partial}{\partial t}\right) \frac{\partial \theta(x, h / 2, t)}{\partial z}, \quad \frac{\partial \theta(x,-h / 2, t)}{\partial z}=0 .
$$

Applying (10) and (13), (31) yields

$$
\frac{\mathrm{d} \bar{\theta}}{\mathrm{d} z}\left(x, \frac{h}{2}, s\right)=\frac{q_{0}}{k s \bar{\Gamma}_{1}}, \quad \frac{\mathrm{d} \bar{\theta}}{\mathrm{d} z}\left(x,-\frac{h}{2}, s\right)=0 .
$$

The other boundary conditions, (29a) and (29b), hold true in the second application. In view of (16) and (32), the thermal influence of the second problem is given by

$$
\bar{Q}^{*}(s)=\frac{c_{1} q_{0}}{k s \bar{\Gamma}_{1}}
$$

and (30a), (30b), (30c), (30d), (30e), and (30f) hold their forms with replacing $\bar{Q}^{*}$ from (28a) and (28b) to (33).

This completes the solution of the mathematical model in the Laplace transformed domain. In order to obtain the solution of the present application in the physical domain, we firstly apply the well-known formula:

$$
f(t)=\frac{1}{2 \pi i} \int_{c-i \infty}^{c+i \infty} \bar{f}(s) e^{s t} \mathrm{~d} s
$$

to (20), (23) and (24a), and (24b) and use the results of (28a) and (28b), (30a), (30b), (30c), (30d), (30e), and (30f) and (33). Secondly, we adopt a numerical inversion method based on 


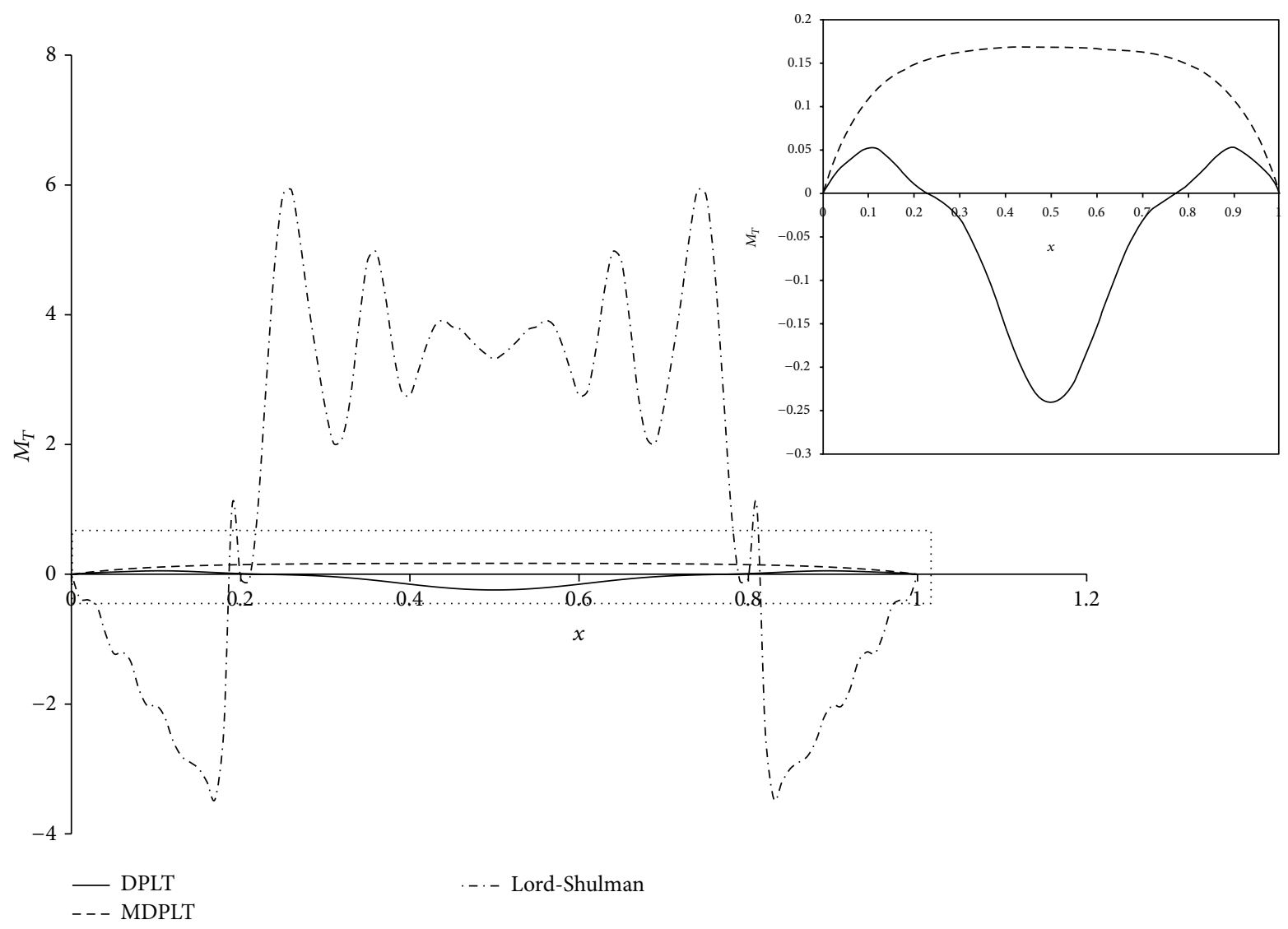

Figure 4: Laser-induced thermal moment at dimensionless time $t=0.1$ under the three models.

the Fourier series expansion, by which the integral (34) can be approximated as a series

$$
\begin{aligned}
f(t)=\frac{e^{c t}}{t_{1}}[ & -\frac{1}{2} \operatorname{Re} \bar{f}(c) \\
& +\sum_{j=0}^{\infty} \operatorname{Re}\left(\bar{f}\left(c+\frac{i j \pi}{t_{1}}\right)\right) \cos \left(\frac{j \pi}{t_{1}}\right) \\
& \left.-\sum_{j=0}^{\infty} \operatorname{Im}\left(\bar{f}\left(c+\frac{i j \pi}{t_{1}}\right)\right) \sin \left(\frac{j \pi}{t_{1}}\right)\right] \\
& -\sum_{j=1}^{\infty} e^{-2 c j t_{1}} f\left(2 j t_{1}+t\right)
\end{aligned}
$$

for $0 \leq t \leq 2 t_{1}$. The above series (35) is called the Durbin formula and the last term in which is called the discretization error. Honig and Hirdes [53] developed a method for accelerating the convergence of the Fourier series and a procedure that computes approximately the best choice of the free parameters. Further, they established a FORTRAN subroutine that helps in implementing series (35). For further numerical methods, see pp. 317-322 in [27].

\section{Numerical Results and Discussion}

The numerical values of the material parameters of gold $(\mathrm{Au})$ are given as follows, see $[23,24,26,54,55]$ :

$$
\begin{gathered}
E=79 \times 10^{9} \mathrm{~Pa} \quad \rho=1930 \mathrm{Kg} \cdot \mathrm{m}^{-3} \quad \nu=0.44 \\
\alpha_{T}=1.4 \times 10^{-5} \mathrm{~K}^{-1} \quad \mathrm{k}=315 \mathrm{~W} \cdot \mathrm{m}^{-1} \cdot \mathrm{K}^{-1} \\
\rho c_{v}=2.492 \times 10^{6} \mathrm{~J} \cdot \mathrm{m}^{-3} \cdot \mathrm{K}^{-1} \quad \tau_{T}=89.286 \mathrm{ps} \\
\tau_{q}=0.7838 \mathrm{ps} \quad \tau_{q_{2}}=0.04 \mathrm{ps} \\
\tau_{m}=0.1725 \mathrm{ps} \quad R=0.93 \\
t_{p}=0.02 \mathrm{ps} \quad J_{0}=3.14 \times 10^{15} \quad \delta=0.1 \mathrm{~h} \\
T_{0}=300 \mathrm{~K} \quad q_{0}=2.25 \times 10^{11} \mathrm{~W} \cdot \mathrm{m}^{-2} .
\end{gathered}
$$

Figure 2 shows the laser-induced lateral deflections at an early instant of time $t=0.1$. A similar graph (for the Lord-Shulman model) has been obtained by Sun et al. [22] at $t=0.4,2$ with the symmetry shape about the axis $x=$ $L / 2$. The DPLT and MDPLT models record values lower than that recorded in the Lord-Shulman model. In the inset plot of Figure 2, the curves inside the dotted rectangle are illustrated describing both the DPLT and MDPLT models of thermomechanics. It is notable that the same result shown 


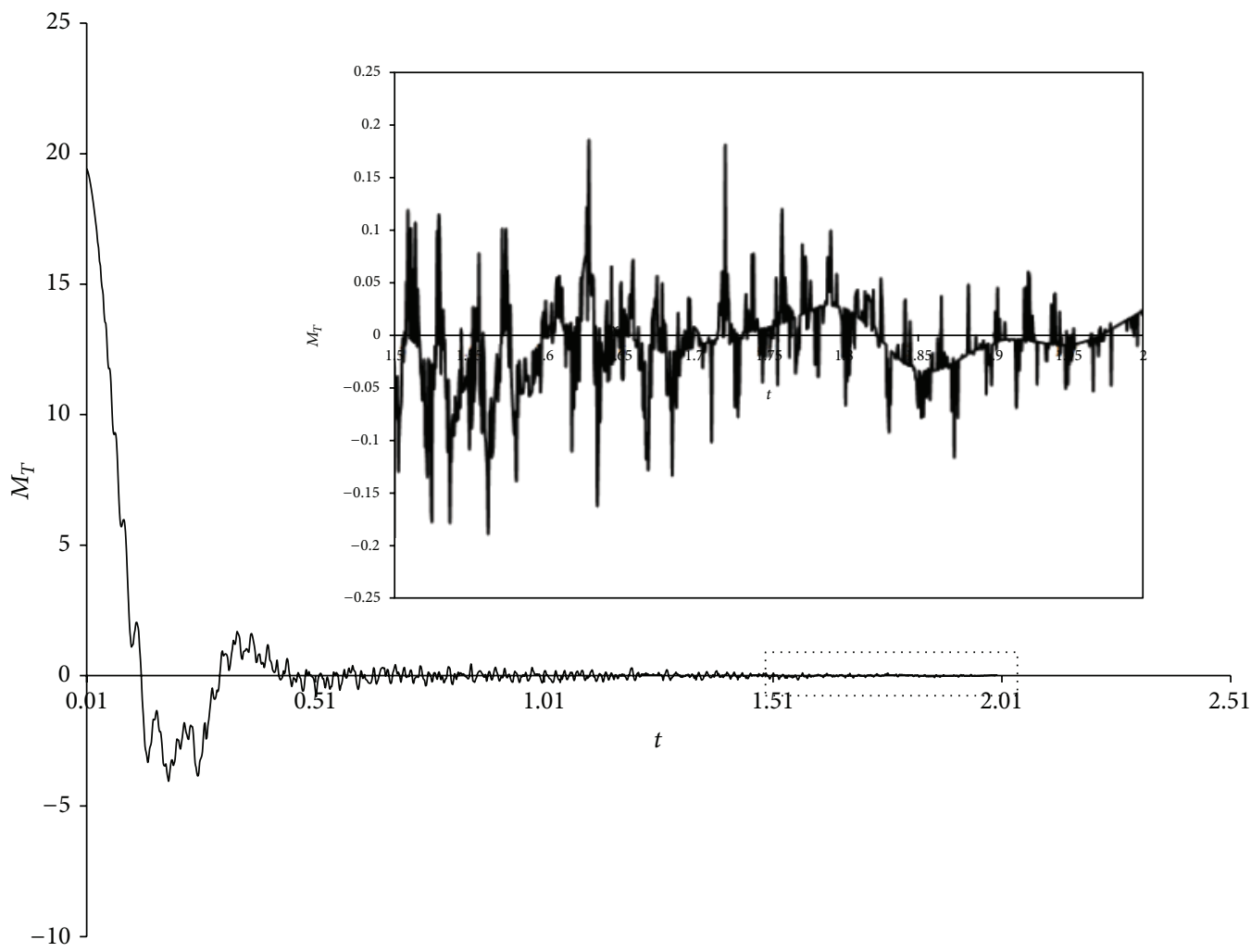

(a)

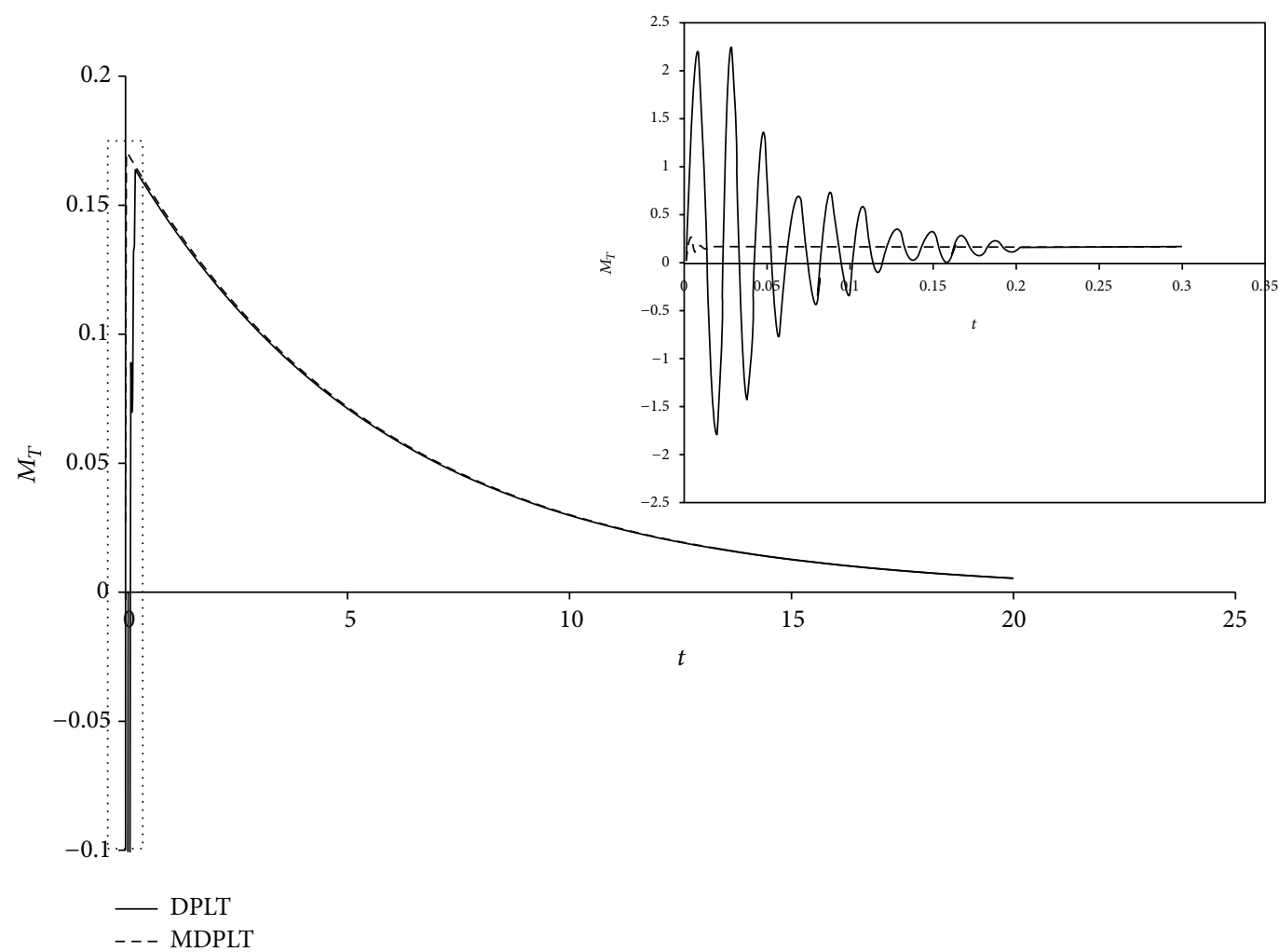

(b)

FIGURE 5: (a) Laser-induced midpoint dimensionless thermal moment under the Lord-Shulman model; see Figure 4 in [22]. (b) Laser-induced midpoint dimensionless thermal moment under the dual-phase-lag models. 


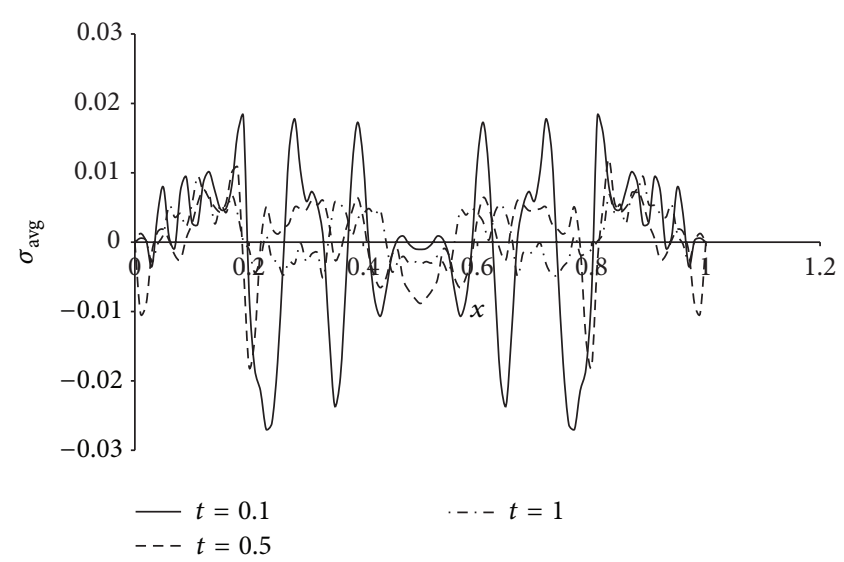

(a)

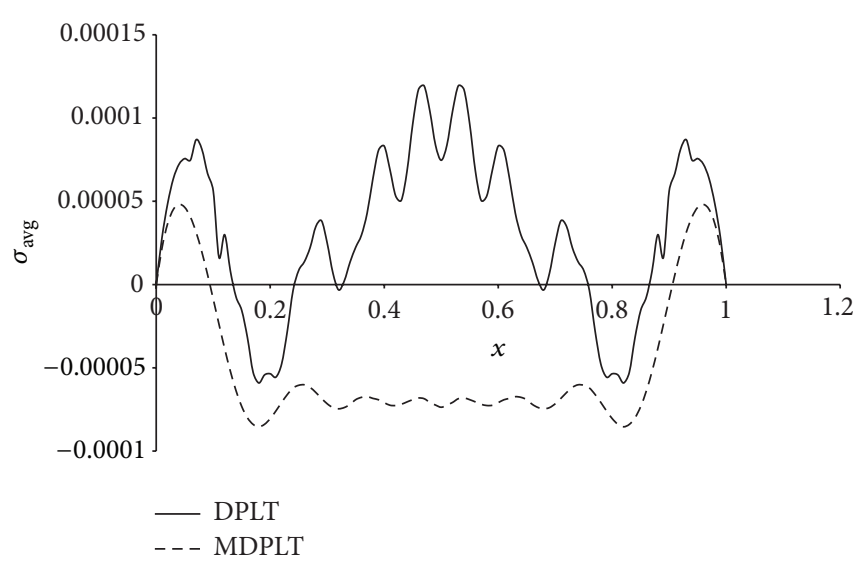

(b)

FIGURE 6: (a) Laser-induced axial stress average at different values of dimensionless time according to the Lord-Shulman model. (b) Laserinduced axial stress at $t=0.1$, according to the dual-phase-lag models.

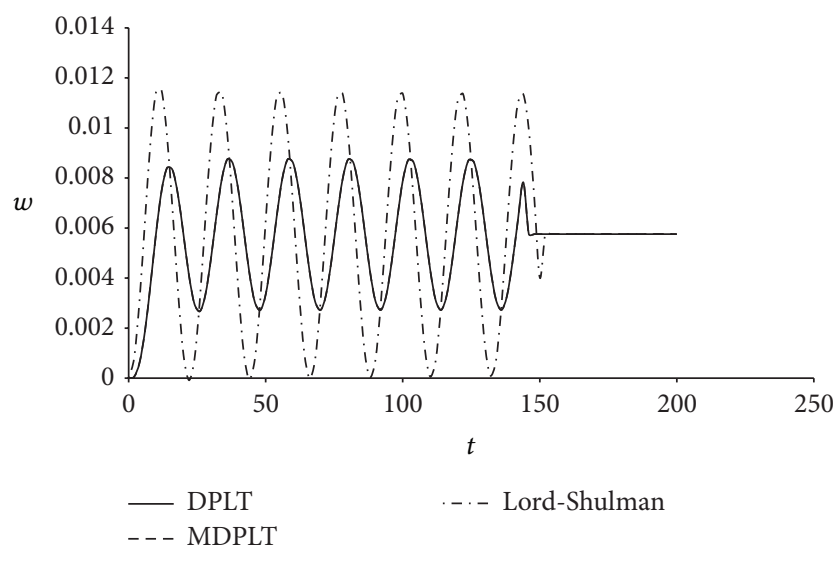

Figure 7: Heat flux-induced midpoint dimensionless lateral deflection under the three models.

in the thermal shock problem, see [26], has been obtained here. Namely, the MDPLT model has a weak effect on the displacement of particles compared with the DPLT model, especially at the small values of time.

Figure 3 focuses on the temporal history of the midpoint vibrations $(x=L / 2)$. Obviously, the Lord-Shulman model predicts thermomechanical waves propagating with velocity faster than the DPLT and MDPLT models, having amplitudes higher than those recorded by the DPLT and MDPLT models. The midpoint returns to the quiescent mode in the LordShulman model before the other models. The inset plot of Figure 3 clarifies the differences between the DPLT and MDPLT lateral deflections. As stated previously [26], the main difference between the DPL and MDPL models appears in the early response times, and the difference between DPLT and MDPLT lateral deflections is apparent in the dimensionless time interval $[0,1]$. The MDPLT model lowers the deflection values and increases smoothly within $[0,0.38]$. This trend is reversed in the subsequent interval $[0.38,0.76]$. DPLT thermomechanical waves propagate with speed faster

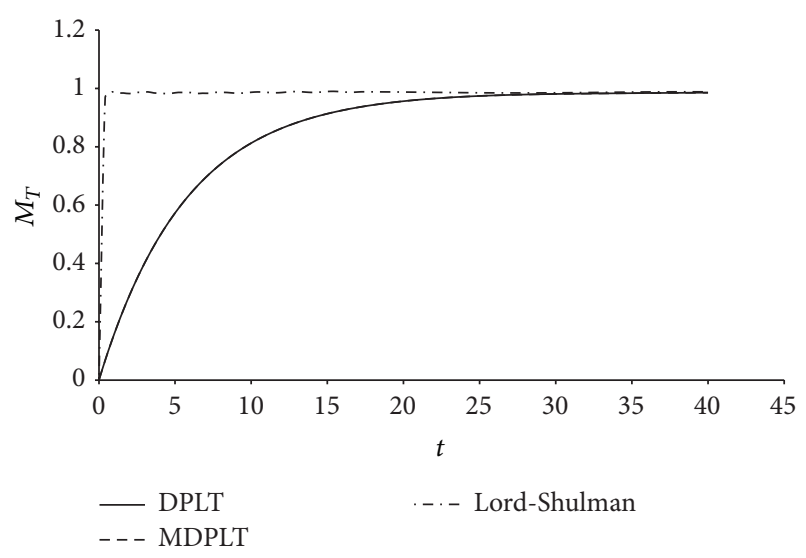

FIgURE 8: Heat flux-induced midpoint dimensionless thermal moment under the three models.

than MDPLT waves if relatively small values of time are considered.

Figures 4 and 5 describe the thermal moment variations with beam length and time. The smooth curves of the DPLT and MDPLT models, which are incomparable with the LordShulman curve, are clear in Figure 4. The DPLT and MDPLT curves in the rectangular frame are illustrated in the inset plot of Figure 4. The difference between DPLT and MDPLT curves drawn in inset-plot 4 is temporarily at $t=0.1$. Inset-plot $5 \mathrm{~b}$ shows the $M_{T}$ vibrations of the midpoint. At $t=0.1$, the two inset-plots 4 and $5 \mathrm{~b}$ coincide; however, in a subsequent instant $t=0.15$, one finds the two curves to reverse their positions near the midpoint, and at $t=0.2$ there is no significant difference between the DPLT and MDPLT thermal moments. Furthermore, in the dimensionless time interval $[0,0.2]$, the thermal moment $M_{T}$ of DPLT model has frequency values higher than that in the MDPLT model. Figure 5(a), actually, supports the current study. It is not dissimilar from Figure 4, which is drawn by Sun et al. [22] for a Silicon beam, except for Figure 5(a), which begins from 


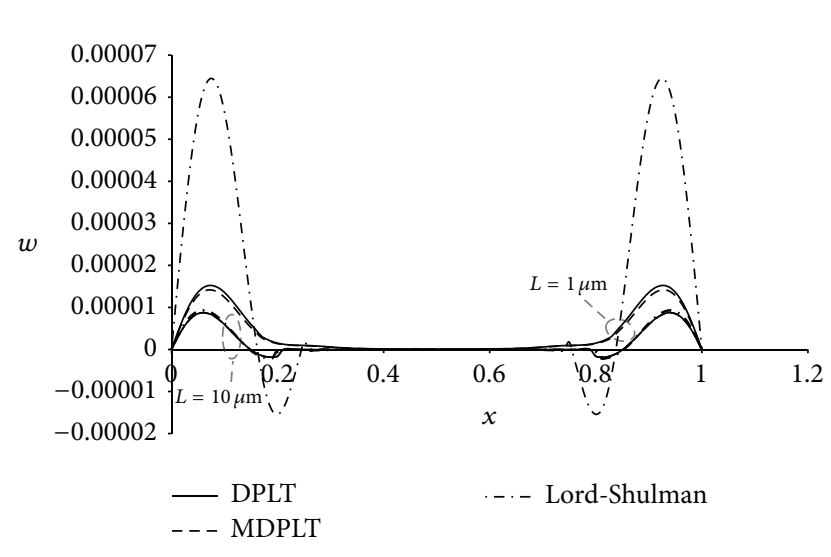

(a)

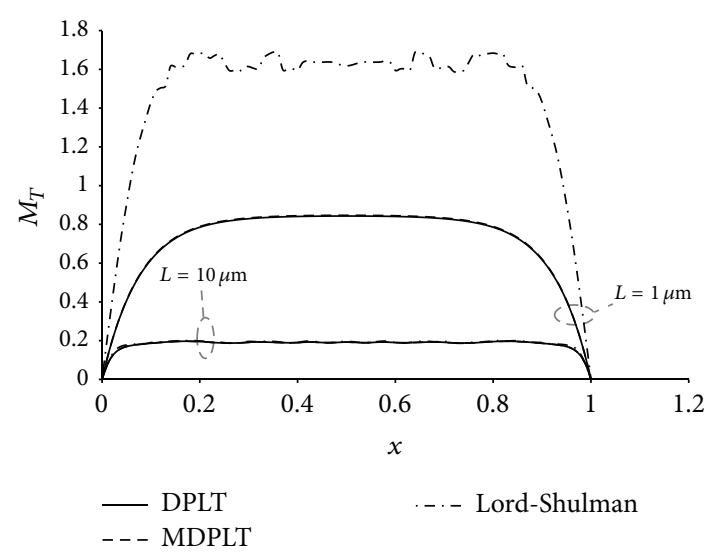

(b)

FIGURE 9: (a) Laser-induced lateral deflection, for a beam with length $L=1$ and $10 \mu \mathrm{m}$, at dimensionless time $t=0.1$ under the three models. (b) Laser-induced thermal moment, for a beam with length $L=1$ and $10 \mu \mathrm{m}$, at dimensionless time $t=0.1$ under the three models.

$t=0.01$, and the decaying rate in the gold beam is faster. It was difficult to draw $M_{T}$ at earlier times than 0.01 . However, it is predicted to begin from null obeying the initial condition. In comparison between Figures 5(a) and 5(b), the DLPT and MDPLT thermal moments have peaks much lower than peaks of Lord-Shulman curve. The midpoint $M_{T}$-variation of LordShulman model has many fluctuations and short time zone of effect, namely, the midpoint thermal moment variation of DPLT and MDPLT models remains for a long time interval. The different velocities of the wave propagation of the three models may be a reason beyond the distinct fluctuations in Figure 5(a) and inset-plot of 5b. The thermal wave velocities of the Lord-Shulman and DPLT and MDPLT models, $C_{\mathrm{LS}}$, $C_{D}$, and $C_{\mathrm{MD}}$, respectively, are given by

$$
\begin{aligned}
& C_{\mathrm{LS}}=\sqrt{\frac{\alpha_{v}}{\tau_{q}}} \quad C_{D}=\sqrt{\frac{2 \alpha_{v} \tau_{T}}{\tau_{q}^{2}}} \\
& C_{\mathrm{MD}}=\sqrt{\frac{\alpha_{v} \tau_{T}}{\tau_{q_{1}} \tau_{q_{2}}}} \quad \alpha_{v}=\frac{k}{\rho c_{v}} .
\end{aligned}
$$

For gold $(\mathrm{Au}), C_{\mathrm{LS}}=1.268 \times 10^{4} \mathrm{~m} / \mathrm{s}, C_{D}=1.914 \times 10^{5} \mathrm{~m} / \mathrm{s}$, $C_{\mathrm{MD}}=6.149 \times 10^{5} \mathrm{~m} / \mathrm{s}$; namely, $C_{\mathrm{LS}}<C_{D}<C_{\mathrm{MD}}$.

The thermal stress variations are plotted in Figures 6(a) and 6(b) along the beam. In accordance with the previous results, the stress curve of the Lord-Shulman model recorded peaks higher than that recorded by the DPLT and MDPLT models. In Figure 6(a), the stress curve along the beam under the Lord-Shulman model is plotted at three different instants $t=0.1,0.5$, and 1 . The low values of stress curve of the MDPLT model are clear in Figure 6(b).

In Figures 7 and 8, the temporal history of the midpoint deflection and thermal moment are represented, respectively, for the heat flux problem. The midpoint lateral deflection of the heat flux application vibrates about the dimensionless value $w=0.057$, while it vibrates about the value $w=0$ in the laser application. In the heat flux problem, the DPLT and MDPLT models reach steady state before the Lord-Shulman model in the heat flux application unlike what is obtained in the laser problem. The history of the midpoint thermal moment variation is represented in Figure 8 for the three models. The sudden increase in the Lord-Shulman curve and the gradual increase in the DPLT and MDPLT curves are characteristic features of the constant heat flux application.

Figures 9(a) and 9(b) show the effect of the beam length on the lattice variables, deflection, and thermal moment, for the laser application. But the result can be generalized to the second application. It can be readily seen from Figures 9(a) and 9(b) that (i) the longer the beam is the lower the lateral deflection and thermal moment are. (ii) The longer the beam is the less difference between models is.

\section{Conclusion}

The macroscopic vibrations of a gold Euler-Bernoulli thermoelastic rectangular microscale beam have been studied during two different ways of heating, the laser-pulse heating, and the constant heat flux on the upper surface. The direct approach method has been employed to obtain the exact solutions in the Laplace transformed domain. A numerical technique has been adopted to recover the solutions in the physical domain. The paper focuses on the distinguishable features of the Lord-Shulman model of thermomechanics, based on the Cattaneo-Vernotte law, and the Tzou-model of thermomechanics (DPLT and MDPLT), based on the QiuTien hyperbolic two-step model.

The dual-phase-lag models (DPLT and MDPLT), basically, depend on a certain mechanism of heat transport. It subdivides the heat transport to three processes $[20,21]$ : (i) the deposition of radiation energy on electron, (ii) the transport of energy by electron subsystem, and (iii) the heating of material lattice via electron-phonon interactions. The single relaxation time model (Lord-Shulman), on the other hand, neglects the electron-phonon interactions during the fast transient processes. This may be the major reason beyond the behavior of DPLT and MDPLT. The results of this 
paper can be supported by the evident differences between the electron and lattice temperatures during ultrafast laser heating; see Figures 1 and 9 in Chen et al. [54] and Figure 2 in Tzou and Pfautsch [55]. There is a material range of picoseconds needed to reach the thermal equilibrium between phonons and electrons (thermalization time), whilst both the electrons and the lattice are heated simultaneously in the Lord-Shulman thermomechanics. Upon reaching the thermal equilibrium between electrons and the lattice, the DPLT and MDPLT become immaterial. Furthermore, as seen from Figures 4, 5(a), and 5(b), the meaningless V-shape has disappeared from the DPLT models, which indicates the improved numerical results of the DPLT models.

One can conclude that whenever the response time is relatively short and the material dimensions are relatively small, the dual-phase-lag thermoelastic models could not be disregarded and could be considered as the most close macroscopic approach to study the general behavior (but not accurate because of the constant thermophysical properties) rather than encountering the strong nonlinearity of the ultrafast model [54].

\section{Appendix}

Upon solving (19b), the characteristic roots of the problem are given by

$$
\begin{aligned}
& \lambda_{1}=\frac{1}{\sqrt{3}}\left\{\ell+2 \kappa_{1} \cos \left(\frac{\cos ^{-1}\left(\kappa_{2}\right)}{3}\right)\right\}^{1 / 2}, \\
& \lambda_{2}=\frac{1}{\sqrt{3}}\left\{\ell-2 \kappa_{1} \sin \left(\frac{\pi-\sin ^{-1}\left(\kappa_{2}\right)}{3}\right)\right\}^{1 / 2} \\
& \lambda_{3}=\frac{1}{\sqrt{3}}\left\{\ell-2 \kappa_{1} \sin \left(\frac{\sin ^{-1}\left(\kappa_{2}\right)}{3}\right)\right\}^{1 / 2},
\end{aligned}
$$

where

$$
\kappa_{1}=\sqrt{\ell^{2}-3 m}, \quad \kappa_{2}=\frac{2 \ell^{3}-9 \ell m+27 n}{2 \kappa_{1}^{3}} .
$$

The problem coefficients, $A_{i}$ and $B_{i}, i=1,2,3$, are determined through

$$
\begin{gathered}
A_{1}=\frac{\bar{Q}^{*}\left(\lambda_{1}^{2}-\alpha_{1}\right)\left(\lambda_{2}^{2}-\alpha_{1}\right)\left(\lambda_{3}^{2}-\alpha_{1}\right)}{\alpha_{1}^{2} \alpha_{2}\left(\lambda_{1}^{2}-\lambda_{2}^{2}\right)\left(\lambda_{1}^{2}-\lambda_{3}^{2}\right)\left(e^{\lambda_{1}}+1\right)}, \\
A_{2}=\frac{\left(\lambda_{3}^{2}-\lambda_{1}^{2}\right)\left(e^{\lambda_{1}}+1\right)}{\left(\lambda_{2}^{2}-\lambda_{3}^{2}\right)\left(e^{\lambda_{2}}+1\right)} A_{1} \\
A_{3}=\frac{\left(\lambda_{1}^{2}-\lambda_{2}^{2}\right)\left(e^{\lambda_{1}}+1\right)}{\left(\lambda_{2}^{2}-\lambda_{3}^{2}\right)\left(e^{\lambda_{3}}+1\right)} A_{1}, \\
B_{1}=e^{\lambda_{1}} A_{1}, \quad B_{2}=e^{\lambda_{2}} A_{2}, \quad B_{3}=e^{\lambda_{3}} A_{3} .
\end{gathered}
$$

\section{Conflict of Interests}

The authors declare that there is no conflict of interests regarding the publication of this paper.

\section{Acknowledgments}

Alexandria University financial support for this work is acknowledged. The authors are indebted to the referees for their helpful comments.

\section{References}

[1] P. M. Morse and H. Feshbach, Methods of Theoretical Physics, McGraw-Hill, New York, NY, USA, 1st edition, 1953.

[2] C. Cattaneo, "A form of heat conduction equation which eliminates the Paradox of instantaneous propagation," Comptes Rendus, vol. 247, pp. 431-433, 1958.

[3] P. Vernotte, "Les paradoxes de la théorie continue de l'équation de la chaleur," Comptes Rendus, vol. 246, pp. 3154-3155, 1958.

[4] P. Vernotte, "Some possible complications in the phenomena of thermal conduction," Comptes Rendus, vol. 252, pp. 2190-2191, 1961.

[5] D. D. Joseph and L. Preziosi, "Heat waves," Reviews of Modern Physics, vol. 61, no. 1, pp. 41-73, 1989.

[6] D. Y. Tzou, "Thermal shock phenomena under high-rate response in solids," in Annual Review of Heat Transfer, C.-L. Tien, Ed., pp. 111-185, Hemisphere Publishing, Washington, DC, USA, 1992.

[7] H. W. Lord and Y. Shulman, "A generalized dynamical theory of thermoelasticity," Journal of the Mechanics and Physics of Solids, vol. 15, no. 5, pp. 299-309, 1967.

[8] M. A. Biot, "Thermoelasticity and irreversible thermodynamics," Journal of Applied Physics, vol. 27, no. 3, pp. 240-253, 1956.

[9] R. S. Dhaliwal and H. H. Sherief, "Generalized thermoelasticity for anisotropic media," Quarterly of Applied Mathematics, vol. 38, no. 1, pp. 1-8, 1980.

[10] H. H. Sherief, "Fundamental solution of the generalized thermoelastic problem for short times," Journal of Thermal Stresses, vol. 9, no. 2, pp. 151-164, 1986.

[11] M. N. Anwar and H. H. Sherief, "State space approach to generalized thermoelasticity," Journal of Thermal Stresses, vol. 11, no. 4, pp. 353-365, 1988.

[12] D. S. Chandrasekharaiah, "Hyperbolic thermoelasticity: a review of recent literature," Applied Mechanics Reviews, vol. 51, no. 12, pp. 705-729, 1998.

[13] R. B. Hetnarski and J. Ignaczak, "Generalized thermoelasticity," Journal of Thermal Stresses, vol. 22, no. 4, pp. 451-476, 1999.

[14] J. Ignaczak and M. Ostoja-Starzewski, Thermoelasticity with Finite Wave Speeds, Oxford Mathematical Monographs, Oxford University Press, Oxford, UK, 2010.

[15] M. I. Kaganov, I. M. Lifshitz, and L. V. Tanatarov, "Relaxation between electrons and crystalline lattices," Soviet Physics JETP, vol. 4, pp. 173-178, 1957.

[16] S. I. Anisimov, B. L. Kapeliovich, and T. L. Perel'man, "Electron emission from metal surfaces exposed to ultra-short laser pulses," Soviet Physics JETP, vol. 39, pp. 375-377, 1974.

[17] S. D. Brorson, J. G. Fujimoto, and E. P. Ippen, "Femtosecond electronic heat-transport dynamics in thin gold films," Physical Review Letters, vol. 59, no. 17, pp. 1962-1965, 1987.

[18] S. D. Brorson, A. Kazeroonian, J. S. Moodera et al., "Femtosecond room-temperature measurement of the electron-phonon coupling constant in metallic superconductors," Physical Review Letters, vol. 64, no. 18, pp. 2172-2175, 1990. 
[19] H. E. Elsayed-Ali, T. Juhasz, G. O. Smith, and W. E. Bron, "Femtosecond thermoreflectivity and thermotransmissivity of polycrystalline and single-crystalline gold films," Physical Review B, vol. 43, no. 5, pp. 4488-4491, 1991.

[20] T. Q. Qiu and C. L. Tien, "Short-pulse laser heating on metals," International Journal of Heat and Mass Transfer, vol. 35, no. 3, pp. 719-726, 1992.

[21] T. Q. Qiu and C. L. Tien, "Heat transfer mechanisms during short-pulse laser heating of metals," Journal of Heat Transfer, vol. 115, no. 4, pp. 835-841, 1993.

[22] Y. Sun, D. Fang, M. Saka, and A. K. Soh, "Laser-induced vibrations of micro-beams under different boundary conditions," International Journal of Solids and Structures, vol. 45, no. 7-8, pp. 1993-2013, 2008.

[23] D. Y. Tzou, "The generalized lagging response in small-scale and high-rate heating," International Journal of Heat and Mass Transfer, vol. 38, no. 17, pp. 3231-3240, 1995.

[24] D. Y. Tzou, "Unified field approach for heat conduction from macro- to micro-scales," Journal of Heat Transfer, vol. 117, no. 1, pp. 8-16, 1995.

[25] D. Y. Tzou and J. Xu, "Nonequilibrium transport: the lagging behavior," in Advances in Transport Phenomena, L. Q. Wang, Ed., pp. 93-170, Springer, Berlin, Germany, 2011.

[26] E. Awad, "On the generalized thermal lagging behavior: refined aspects," Journal of Thermal Stresses, vol. 35, no. 4, pp. 293-325, 2012.

[27] D. Y. Tzou, Macro- to Microscale Heat Transfer: The Lagging Behavior, Taylor and Francis, New York, NY, USA, 1997.

[28] M. A. Abdou, I. H. El-Sirafy, and E. Awad, "Thermoelectric effects of metals within small-scale regimes: theoretical aspects," Chinese Journal of Physics, vol. 51, no. 5, pp. 1051-1066, 2013.

[29] J. P. Jones, "Thermoelastic vibrations of a beam," Journal of the Acoustical Society of America, vol. 39, no. 3, pp. 542-548, 1966.

[30] J. Ignaczak and W. Nowacki, "Transversal vibration of a plate, produced by heating," Archiwum Mechaniki Stosowanej, vol. 13, pp. 651-667, 1961.

[31] C. V. Massalas and V. K. Kalpakidis, "Coupled thermoelastic vibrations of a simply supported beam," Journal of Sound and Vibration, vol. 88, no. 3, pp. 425-429, 1983.

[32] B. V. Sankar and J. T. Tzeng, "Thermal stresses in functionally graded beams," AIAA Journal, vol. 40, no. 6, pp. 1228-1232, 2002.

[33] B. V. Sankar, "An elasticity solution for functionally graded beams," Composites Science and Technology, vol. 61, no. 5, pp. 689-696, 2001.

[34] M. H. Babaei, M. Abbasi, and M. R. Eslami, "Coupled thermoelasticity of functionally graded beams," Journal of Thermal Stresses, vol. 31, no. 8, pp. 680-697, 2008.

[35] M. R. Eslami and H. Vahedi, "Coupled thermoelasticity beam problems," AIAA journal, vol. 27, no. 5, pp. 662-665, 1989.

[36] Y. Sun, D. Fang, and A. K. Soh, "Thermoelastic damping in micro-beam resonators," International Journal of Solids and Structures, vol. 43, no. 10, pp. 3213-3229, 2006.

[37] Z. F. Khisaeva and M. Ostoja-Starzewski, "Thermoelastic damping in nanomechanical resonators with finite wave speeds," Journal of Thermal Stresses, vol. 29, no. 3, pp. 201-216, 2006.

[38] H. M. Youssef and K. A. Elsibai, "Vibration of nano beam induced by Ramp type heating," World Journal of Nano Science and Engineering, vol. 1, pp. 37-44, 2011.

[39] H. M. Youssef and K. A. Elsibai, "Vibration of gold nanobeam induced by different types of thermal loading-a state-space approach," Nanoscale and Microscale Thermophysical Engineering, vol. 15, no. 1, pp. 48-69, 2011.

[40] K. A. Elsibai and H. M. Youssef, "State-space approach to vibration of gold nano-beam induced by ramp type heating without energy dissipation in femtoseconds scale," Journal of Thermal Stresses, vol. 34, no. 3, pp. 244-263, 2011.

[41] H. M. Youssef, "Vibration of gold nanobeam with variable thermal conductivity: state-space approach," Journal of Applied Nanoscience, 2013.

[42] I. H. el-Sirafy and M. A. Abdou, "First and second fundamental problems of infinite plate with a curvilinear hole," Journal of Mathematical and Physical Sciences, vol. 18, no. 2, pp. 205-214, 1984.

[43] M. A. Abdou and S. A. Aseeri, "Closed forms of goursat functions in presence of heat for curvilinear holes," Journal of Thermal Stresses, vol. 32, no. 11, pp. 1126-1148, 2009.

[44] S. Chiriţă and R. Quintanilla, "Spatial decay estimates of SaintVenant type in generalized thermoelasticity," International Journal of Engineering Science, vol. 34, no. 3, pp. 299-311, 1996.

[45] E. S. Awad, "A note on the spatial decay estimates in non-classical linear thermoelastic semi-cylindrical bounded domains," Journal of Thermal Stresses, vol. 34, no. 2, pp. 147-160, 2011.

[46] B. Carbonaro and R. Russo, "Energy inequalities and the domain of influence theorem in classical elastodynamics," Journal of Elasticity, vol. 14, no. 2, pp. 163-174, 1984.

[47] E. Awad, "Domain of influence theorem and uniqueness results in magneto-thermoelasticity," International Journal of Applied Electromagnetics and Mechanics, vol. 37, no. 1, pp. 1-8, 2011.

[48] R. B. Hetnarski and M. R. Eslami, Thermal Stresses-Advanced Theory and Applications, vol. 32, Springer, New York, NY, USA, 2009.

[49] L. Y. Bahar and R. B. Hetnarski, "Direct approach to thermoelasticity," Journal of Thermal Stresses, vol. 2, no. 1, pp. 135-147, 1979.

[50] M. A. Ezzat and E. S. Awad, "Micropolar generalized magnetothermoelasticity with modified Ohm's and Fourier's laws," Journal of Mathematical Analysis and Applications, vol. 353, no. 1, pp. 99-113, 2009.

[51] M. A. Ezzat and E. S. Awad, "Constitutive relations, uniqueness of solution, and thermal shock application in the linear theory of micropolar generalized thermoelasticity involving two temperatures," Journal of Thermal Stresses, vol. 33, no. 3, pp. 226250, 2010.

[52] D. Y. Tzou, "Experimental support for the lagging behavior in heat propagation," Journal of Thermophysics and Heat Transfer, vol. 9, no. 4, pp. 686-693, 1995.

[53] G. Honig and U. Hirdes, "A method for the numerical inversion of Laplace transforms," Journal of Computational and Applied Mathematics, vol. 10, no. 1, pp. 113-132, 1984.

[54] J. K. Chen, J. E. Beraun, and D. Y. Tzou, "Thermomechanical response of metals heated by ultrashort-pulsed lasers," Journal of Thermal Stresses, vol. 25, no. 6, pp. 539-558, 2002.

[55] D. Y. Tzou and E. J. Pfautsch, "Ultrafast heating and thermomechanical coupling induced by femstosecond lasers," Journal of Engineering Mathematics, vol. 61, no. 2-4, pp. 231-247, 2008. 


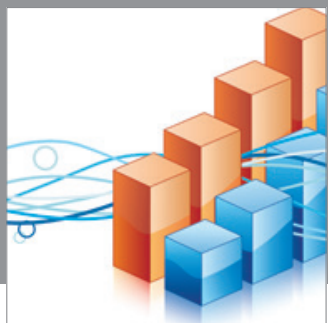

Advances in

Operations Research

mansans

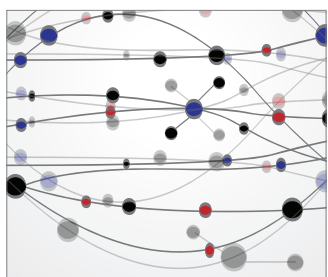

The Scientific World Journal
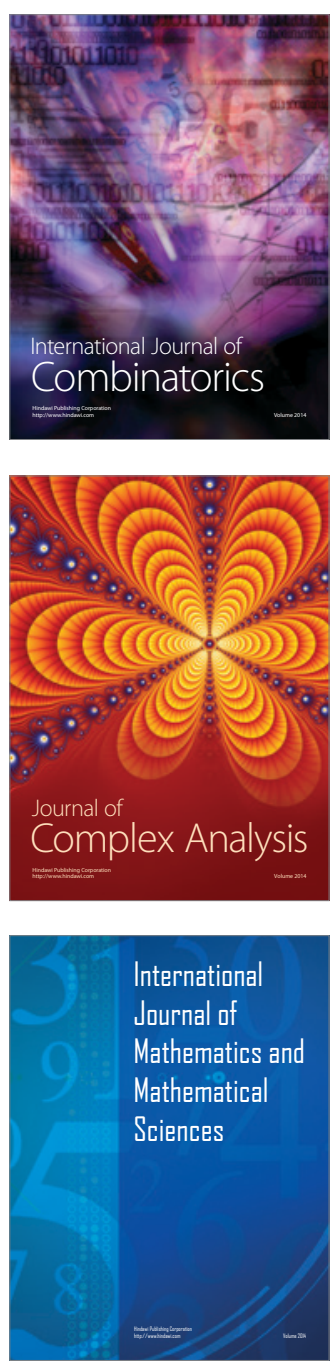
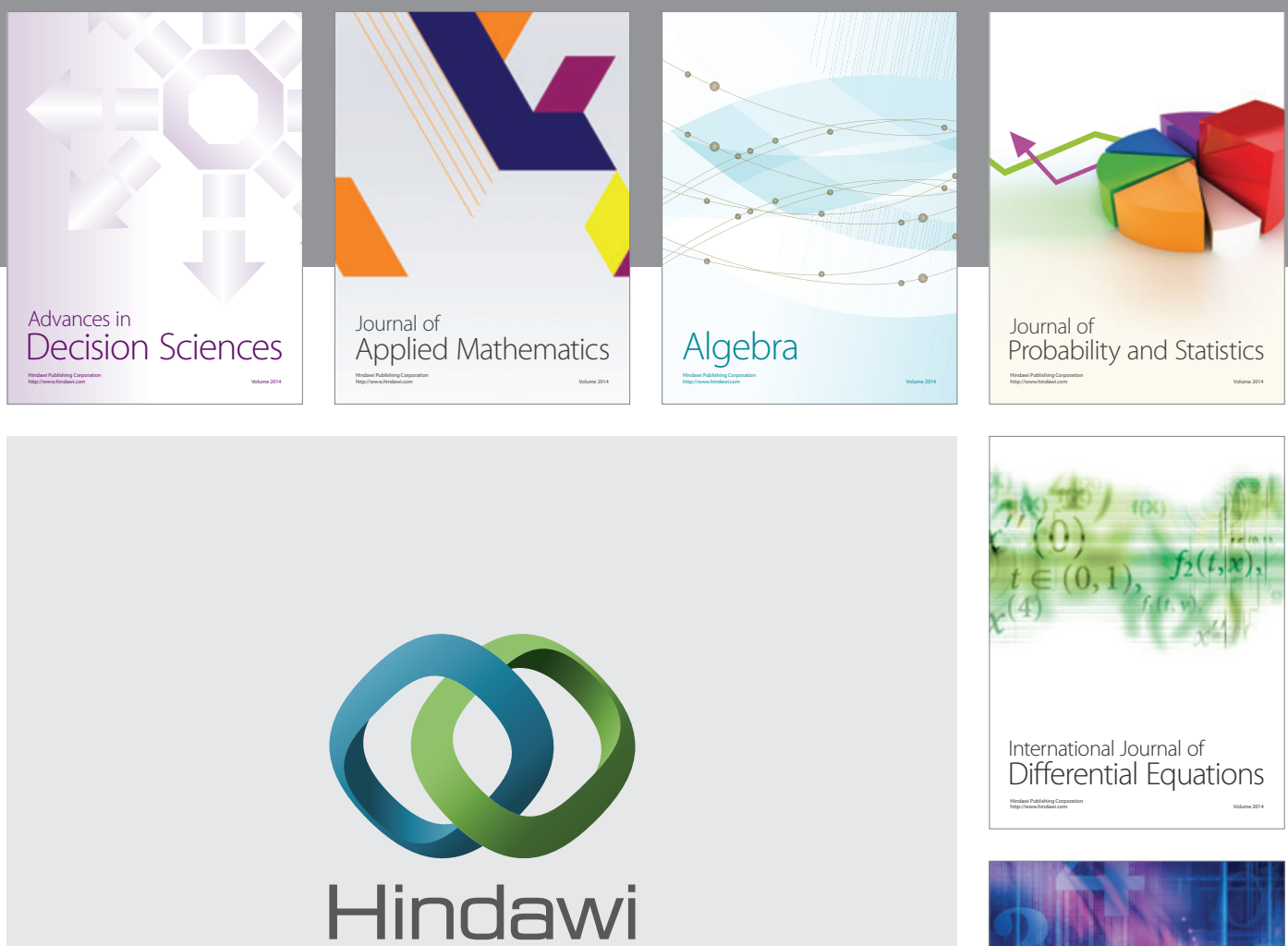

Submit your manuscripts at http://www.hindawi.com
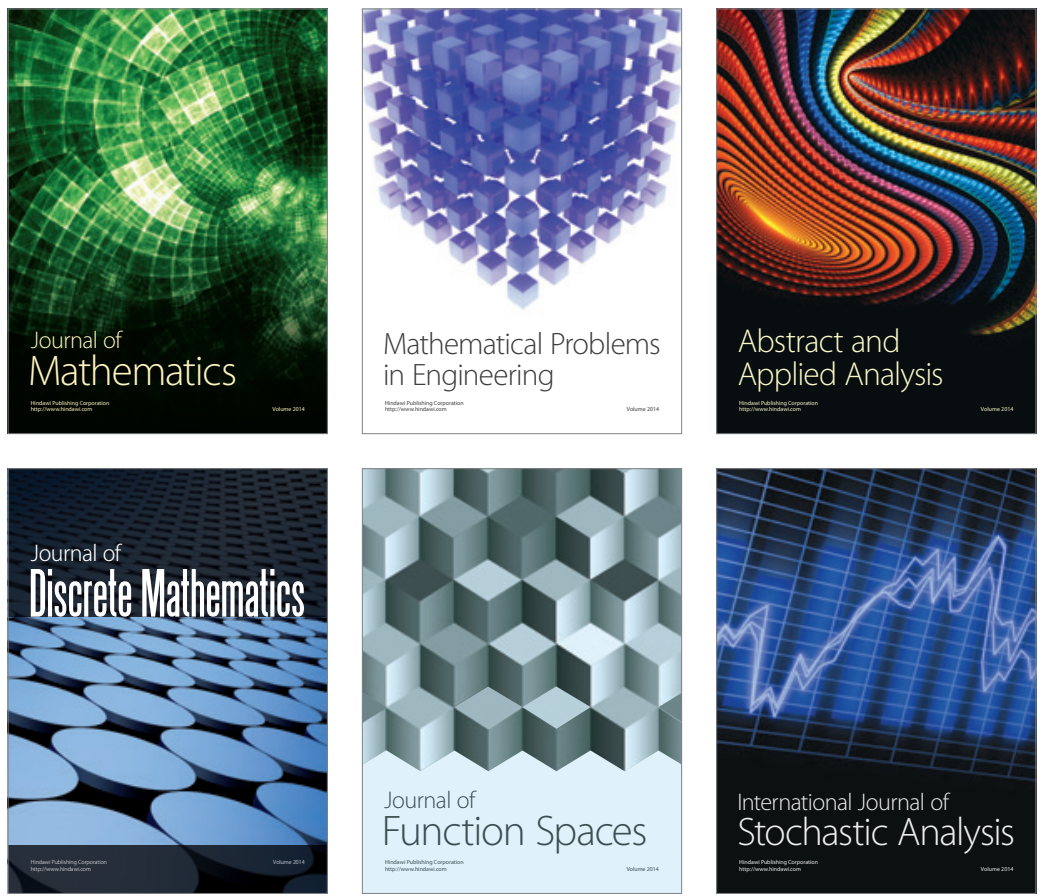

Journal of

Function Spaces

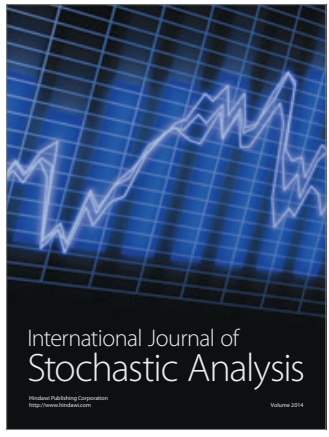

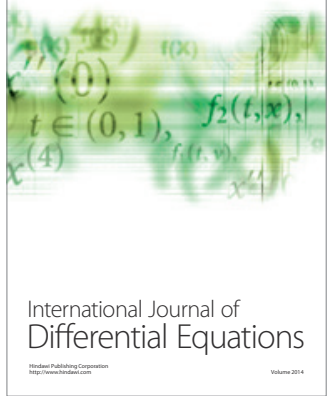
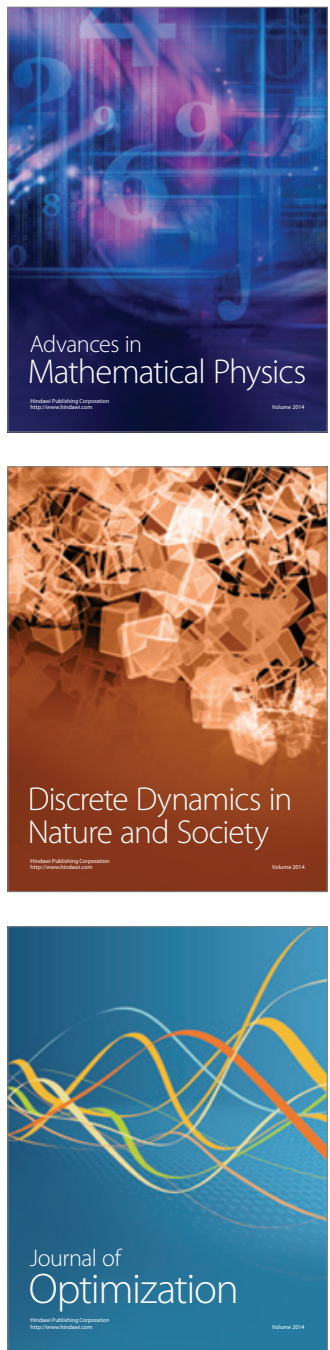